\title{
Positive thinking and physical activity motivation for one individual with multiple sclerosis: A qualitative case-study
}

Samuel M. Hall-McMaster BSC (hons)

Student, Department of Psychology, University of Otago

Gareth J. Treharne PhD, AFBPSS

Senior Lecturer, Department of Psychology, University of Otago

Catherine M. Smith DipPhty, PhD

Research Fellow, Centre for Health, Activity and Rehabilitation Research, School of Physiotherapy, University of Otago

\section{ABSTRACT}

Regular physical activity (PA) is known to benefit individuals with multiple sclerosis (MS) but people with MS tend to do less PA than the general population. Thought processes such as positive thinking may help to explain PA motivation among individuals with MS. The present study investigated thoughts about PA motivation in one man with MS (aged 70 years; pseudonym Norman). We asked Norman to think aloud while completing standardised measures of PA, stages of change, self-efficacy and in response to planned and spontaneous questions. The data were subjected to inductive thematic analysis and two major themes were formulated: positive thinking as Norman's way to fight against MS; and goals give a positive purpose to Norman's engagement in PA. Theme one consisted of three subthemes: coping with MS by choosing to think positively; using positivity to maintain control; and using PA to think positively. Theme two consisted of two subthemes: viewing PA as a necessity for goal achievement; and goals providing determination. Norman's case has implications for enhancing future physiotherapy interventions. In particular, addressing positive thinking and purposeful goal setting may help physiotherapists to increase PA motivation in individuals with MS and thereby encourage more regular engagement in PA.

Hall-McMaster S M, Treharne G J, Smith C M (2016) Positive thinking and physical activity motivation for one individual with multiple sclerosis: A qualitative case-study New Zealand Journal of Physiotherapy 44(1): 26-32. doi: 10.15619/NZJP 144.1.04

Keywords: Physical activity, Motivation, Qualitative research, Case-study, Multiple sclerosis

\section{INTRODUCTION}

Regular physical activity (PA) is beneficial for people with multiple sclerosis (MS) and has the potential to reduce the physical impact of MS after three months (Hale et al 2013, Learmonth et al 2013). In the longer term, people with MS doing regular PA report less fatigue, fewer symptoms of depression and a greater quality of life (Stroud and Minahan 2009). Despite these benefits, people with MS tend to be less active than the general population (Motl et al 2005). Thus, it is important to understand what motivates people with MS on a case by case basis so physiotherapists and other healthcare professionals can help individuals with MS engage in regular PA over the long-term.

Individuals with MS are motivated to do PA for a variety of reasons. For men with MS, goal readjustment is used to overcome the impact of fatigue on PA levels (Smith et al 2014). Men and women with MS may also be motivated to exercise as a means of cultivating optimism and hope (Kasser 2009).

Thought processes such as positive thinking may provide an avenue for enhancing PA motivation for individuals with MS. Kosma et al (2012) argued PA interventions should first emphasise cognitive processes of change such as positive thoughts about PA and then be followed by behavioural strategies like goal setting.
Indeed long-term engagement in PA may be influenced by particular thoughts. According to Smith (2012), the positive thinking inherent in self-efficacy may influence enjoyment of exercise and thus long-term exercise engagement. In addition, Morrison and Stuifbergen (2014) found social and physical outcome expectations explained $11.5 \%$ of the variance in PA participation among individuals with long-standing MS (>15 years). They concluded positive social and physical outcome expectations may enhance PA motivation in people with longstanding MS. Hence, thought-based practices may provide a positive addition to current strategies to help people with MS increase motivation for PA. However, there is an unaddressed need to investigate the kinds of thoughts that affect motivation for PA among individual cases with MS.

Case study methodology provides useful insights into the experiences of unique individuals (Radley and Chamberlain 2001) that can inform further research into ways of potentially improving practice. In addition, the concurrent think-aloud method, in which thought processes and responses to questions are verbalised, can provide substantial insight into a participant's perspective (Lundgrén-Laine and Salanterä 2010).

The aim of the present study was to explore thoughts around PA motivation in one man with MS who took part in a larger qualitative study, which used a think-aloud framework (Hall- 
McMaster et al 2015). The participant stood out based on his relatively high level of impairment, and description of thinking positively. A case-study approach allowed us to further understand how positive thinking may relate to PA motivation within his particular context (Willig 2008). In addition, it enabled an in-depth exploration of this one individual's experience, revealing thoughts and processes that might apply to others (Radley and Chamberlain 2001). Our main research question was: How does this person with MS describe his positive thinking related to PA and his motivations to be physically active, despite having a high level of physical impairment?

\section{METHODS}

\section{Participant}

We gave the pseudonym Norman to our case-study participant Norman was diagnosed with MS 5 years prior to the interview and had been experiencing symptoms for 8 years. At the time of the interview in December 2013 Norman was 70 years old and dependent on an electric wheelchair for mobility. He identified as straight, New Zealand Pākehā and had been married for 49 years. The study was approved by the University of Otago Human Ethics Committee. Norman was recruited through an exercise class at the University of Otago's School of Physiotherapy and signed informed consent before taking part.

\section{Design}

In this article we describe an in-depth qualitative analysis of a single case, which focuses on our interpretation of Norman's experience (Braun and Clarke 2013, Radley and Chamberlain 2001, Koro-Ljungberg et al 2013, Thomas 2010, Willig 2008). We used the concurrent think-aloud method, a valuable method in health research (Adamson et al, 2004), to gather qualitative and quantitative data, and understand Norman's perspectives in-depth (Lundgrén-Laine and Salanterä 2010). The think-aloud method is appropriate for a case-study (Fonteyn et al 1993) in which the focus is on in-depth understanding, rather than prevalence of certain perspectives (McGavock and Treharne 2011). We utilised triangulation (the integration of various sources) to better understand Norman's experience by considering how quantitative data (his responses to items in standardised questionnaires) compared to qualitative findings (his think-aloud responses). In accordance with Willig's (2008) typology our case-study design was instrumental, with an interest in the phenomena of positive thinking and PA motivation; explanatory, considering Norman's case in the context of existing theoretical frameworks; and pragmatic because our central research question guided data collection and analysis.

\section{Procedure}

We made use of Norman's data from a single interview session. Norman completed a series of standardised questionnaire measures during a concurrent think-aloud procedure, in which he verbalised his thought processes while reading items and responding to questions. The think-aloud approach has been widely used in health psychology (see Al-Janabi et al 2013, Anderson-Lister and Treharne 2014), to explore perceptions of people undergoing physiotherapy (Van Oort et al 2011) and attempting to increase PA levels (French et al 2007) because it gains substantial insight into participants' perspectives
(Lundgrén-Laine and Salanterä 2010, Koro-Ljungberg et al 2013, McGavock and Treharne 2011). To date, in the field of MS, the think-aloud method has only been used to assess the content validity of new questionnaires (Wicks et al 2012). In the present study, we took a semi-structured approach to the think-aloud interview by using a combination of a standardised set of questionnaires, a series of 11 planned verbal questions (details available upon request from the corresponding author) and further questions asked as needed to reach the desired depth in our qualitative data. The interview lasted one hour and 16 minutes. It was audio recorded and transcribed by an independent company under a confidentiality agreement.

Measures

Six questionnaire measures were used in the following order:

Demographics. Questions about age, gender identity, sexual orientation, relationship status, living situation, ethnicity, qualifications and job status. The date MS symptoms first appeared and the date of MS diagnosis were also requested.

A diary of self-reported PA from the previous day (Hale et al 2013). Norman's most active type of PA from the previous day formed the basis for a series of planned verbal questions exploring his feelings, thoughts and motivations for that specific activity.

Barriers and strategies for physical activity (BSPA) (Hale et al 2013). Twelve PA barriers and 12 strategies for overcoming barriers (answered 'No', 'Maybe' or 'Definitely').

Stages of change for physical activity (Plotnikoff et al 2007). One question using the definition of 'Physical activity' as doing activities at a moderate intensity, such as brisk walking and 'Regular physical activity' as doing a total of 30 minutes of PA (or more) on a given day, three or more days every week.

Stages of change for positive thinking (adapted from Plotnikoff et al 2007). One question using definitions of 'positive thinking' as focusing on the positive aspects of a situation, including the use of uplifting thoughts, images or sounds and 'choosing to think positively' as deliberately bringing these to mind for five minutes or more, without interruption, three or more days each week (based on Bekhet and Zauszniewski 2013).

The MS Self-efficacy Scale (MSSS) (Rigby et al 2003). Fourteen questions about control over quality of life specific to MS, with two additional questions in this study: "I deliberately choose to think positively in order to help me cope with my illness" and "I choose to take control of how I interpret my MS symptoms". A higher MSSS score indicates higher self-efficacy.

\section{Analysis}

The qualitative data from Norman's think-aloud interview were analysed using inductive thematic analysis based on the steps outlined by Braun and Clarke $(2006,2013)$. The themes formulated were data-driven. Analysis was conducted under the epistemology of critical realism and at a semantic level, based on surface word meanings (Braun and Clarke 2006, 2013). Norman's transcript was repeatedly read by the first author and initial impressions were noted. The transcript was systematically coded for content relating to the research questions. Codes were collated into candidate themes/subthemes for Norman 
as a single case and then critically reviewed by systematically re-reading extracts coded under each theme. The themes were named and data relating to each theme were discussed by the researchers. Finally, the following results section was produced, outlining the two major themes formulated from Norman's qualitative data. Supporting quotes are presented and questionnaire scores on the standardised measures are used to provide context and triangulation (Treharne and Riggs 2014). Within quotes any clarifications are contained in square brackets. Short pauses are indicated by ellipses and short edits are indicated by ellipses within square brackets. Italicised sections indicate where Norman was directly reading items from the questionnaires.

\section{RESULTS}

Theme one: Positive thinking as Norman's way to fight against MS

Subtheme 1.1: Coping with MS by choosing to think positively

Norman expressed the importance of positive thinking as a means to cope with his illness. Upon receiving his diagnosis he chose to see his MS optimistically and has "been positive ever since". This positivity requires Norman to undertake deliberate cognitive action. His motivation for choosing to think positively is to avoid entering a negative cycle, which might prevent him from feeling good about himself:

I deliberately [think] positively in order to help me cope with my illness [MSSS] ... I think that the big big part about it is positive, um being positive at all times because if you're not positive you go into depression and there's no way I'm going into depression.

On the MSSS, Norman strongly agreed with the italicised statement above. Furthermore, he indicated a relatively strong disagreement $(2 / 6$, where 1 = strongly disagree) that he sometimes felt inadequate as a person because of his condition. This highlights Norman's positive self-worth, which he indicated helps him cope with his MS.

\section{Subtheme 1.2: Using positivity to maintain a sense of control}

Positivity is a way for Norman to ensure he is not controlled by his MS. Although his MS has confined him to a wheelchair, Norman refuses to let it dictate his life. His ability to remain in control is driven by his positivity and optimism for the future. Norman repeatedly described having a "positive feel" about being able to walk independently, which has allowed him to remain in control of his life:
I often feel that MS controls my life [from the MSSS]... No... well it is controlling that, not being able to walk but um there's a positive feel that I'm gonna walk so ... so I'm not gonna let it you know control, control my life.

Overall, subthemes 1.1 and 1.2 are supported by Norman's MSSS score of 72/84. This score indicates Norman is very high in self-efficacy, supporting his descriptions of coping with MS (subtheme 1.1) and maintaining control over his condition (subtheme 1.2). Furthermore, Norman strongly agreed that he could keep MS from interfering with time with friends and family, that there were things he could do to control his fatigue and that he had as much independence as he felt he needed. All of these statements support the proposal that he has a strong confidence in his ability to cope and a high degree of perceived control.

\section{Subtheme 1.3: Using PA as a means to think positively}

For Norman, PA is one way of maintaining a positive outlook. He described gaining huge satisfaction in doing PA because he knows it is helping him to better his current situation and makes him believe his goals are possible. PA also allows Norman to maintain a positive self-image, as a confident individual who would "attempt anything". The positive thinking generated through Norman's PA flowed onto positive emotional states, reinforcing his efforts and helping motivate him to continue PA:

I would tell myself that I would feel more confident in myself if I were more physically active [from the BSPA].... Well I do feel confident in myself ... it's through the fact the physical activity is keeping me feeling like that, yeah that things are going to get better, so I would say that is a definite.

... and afterwards, yeah you do, you a lot, you feel good with it because you think well what l'd like to be able to do is ah, once they've been done is be able to get up and do some walking.

While PA helps Norman to think positively, positive thinking also helps him to engage in PA. On the BPSA (Hale et al 2013), Norman indicated that deliberate positive thinking strategies would definitely be helpful to overcome PA barriers. In particular, he indicated that self-talk statements, such as 'I am being good to myself by taking care of my body in this way' from the BPSA would be effective. Positive thinking about his future self would also be helpful, as Norman indicated that he would do PA despite barriers because he knows he will feel better afterwards.

\section{Table 1: Themes and subthemes}

\begin{tabular}{llll}
\hline Theme 1 & Subthemes & Theme 2 & Subthemes \\
\hline Positive thinking as Norman's & 1.1 Coping with MS by & Goals give a positive purpose & 2.1 Viewing PA as a necessity \\
way to fight against MS & choosing to think positively & to Norman's PA & 2.2 Goals provide \\
& 1.2 Using positivity to maintain & determination \\
& a sense of control & \\
& 1.3 Using PA as a means to & \\
think positively & \\
\hline
\end{tabular}


Overall, the theme of positive thinking in spite of MS and its relation to PA is supported by the fact that Norman is in a maintenance stage of change for both choosing to think positively and regular PA, having been doing so for more than 6 months at the time of the interview. While this does not imply causation, the data presented above would suggest that, for Norman, the two are very much interlinked.

Theme two: Goals give a positive purpose to Norman's engagement in PA

\section{Subtheme 2.1: Viewing PA as a necessity for goal achievement}

Norman talked about PA being a necessity to make his goal of walking become a reality; to "get the legs moving and walking". Seeing PA as a necessity for his rehabilitation allows Norman to relentlessly pursue his goals, which he described in a similar way to his sense of control (subtheme 1.2). Here his "positive feel" relates to positive thinking about improvements in the future and ultimately a purpose in doing PA:

... I don't stop [...] going to the class ... because I know they're a necessity ... for my recuperation or for me getting up to walk ... that's you know the positive feel I've still got, I'll always have that positive feel there that um, these things, these physical activity are there to help me get better, not for me to sit back and say bugger it.

\section{Subtheme 2.2: Goals provide determination}

Norman showed remarkable determination towards his "ultimate" goal of walking, despite his relatively high level of physical impairment. Seeing PA as a means to help him get there, Norman's motivation for PA has remained high "all the way throughout" his illness. Setbacks, and rare moments of doubt, only increase his motivation to be physically active and his determination to walk again:

I sometimes have thoughts about whether my condition will get worse [from the MSSS] ... Not very often do I think about that ... it just makes me more determined ... what worse could it do to me now, I can't walk ... I wanna walk and that's my determination...

Norman's determination to reach his goals through PA was further supported by his indication of being in the maintenance stages of change for PA. His determination (as well as positivity and optimism) were also highlighted by his indication of experiencing very few barriers to PA, despite being the most physically impaired of the participants in our wider study (HallMcMaster et al 2015). Of 12 possible barriers, Norman indicated only two may apply to him (but do not definitely), which related to cold weather and not feeling like doing PA. However, Norman did not believe barriers like fatigue, friends' expectations, being worried and stressed would affect his PA engagement. This supports Norman's strong sense of self-efficacy, and earlier subthemes around his ability to cope (1.1) and control (1.2).

\section{DISCUSSION}

In the present study, we used a single case-study method to explore Norman's thoughts about his PA motivation. Two major themes were formulated: positive thinking as Norman's way to fight against MS; and goals that give a positive purpose to Norman's engagement in PA. Norman had very high PA motivation and was extremely positive, despite being wheelchair-bound due to his MS. His case demonstrates how positivity and purpose may benefit physiotherapy practice by helping improve PA motivation in individuals with MS.

Theme one was characterised by positivity. For Norman, deliberately thinking positively is critical in coping with the challenges presented by his MS, especially given his relatively high level of impairment (subtheme 1.1). Positivity also allows Norman to retain control over his life (subtheme 1.2). Indeed the importance of control has been previously documented in the MS literature. Smith et al (2011) found control over fatigue influenced exercise choice, while Hale et al (2012) concluded control over PA increases the likelihood of long-term PA engagement. For Norman positivity is one way to achieve a sense of control. Thus control gained through positivity may be useful in encouraging PA engagement for individuals with MS, particularly those who have lost former capabilities. Furthermore, Norman's case suggests that positivity may not only be important when people with MS begin an exercise programme (Smith et al 2009) but also over the long term. Physiotherapists could consider fostering positivity in people with MS using thought strategies, such as positive self-talk, which can be helpful for focusing on the positive aspects of life for people with neurological conditions (Roger et al 2014). For example, at the end of an initial appointment, physiotherapists could ask patients if they would be willing to include positive self-talk in their treatment plan. If patients feel this would be helpful, physiotherapists could then provide a list of self-talk phrases to be repeated 30 times or more throughout the day. Examples of positive self-talk phrases from questionnaires in the present study were 'I deliberately choose to think positively in order to help me cope with my illness' and, in relation to PA, 'I am being good to myself by taking care of my body in this way'. People with MS and physiotherapists may benefit from working together to create personalised self-talk statements about ways to engage in PA and overcome barriers. Thus when PA motivation is required or a barrier is experienced, the patient has a series of phrases they can call upon and repeat to psychologically ready themselves for physical action.

Norman's use of PA as a means to think positively (subtheme 1.3) is consistent with Dlugonski et al's (2012) findings that women with MS are motivated to do PA to feel good and gain a sense of accomplishment. The present case extends these findings by providing evidence these reasons can also apply to men with MS. Using PA to think positively is consistent with Kasser (2009) who found individuals with MS may be motivated to exercise as a means of cultivating optimism and hope. For Norman, PA was critical to maintain optimism and hope about being able to walk in the future, suggesting these forms of positivity may be strong motivators for PA, as well as exercise.

In addition, Norman was motivated to do PA in order to retain a sense of self-confidence. He scored very highly on self-efficacy and was in the maintenance stage both for regular PA and choosing to think positively. In combination, these findings 
are consistent with research by Chiu et al (2012), showing individuals with MS in a more advanced stage of change for PA had higher levels of self-efficacy. Norman's case also shows that high self-efficacy may present itself in a maintenance stage of change for PA, as well as the action stage examined by Chiu et al (2012). Physiotherapists may consider assessing self-efficacy for both PA and wider contexts, using tools such as the MSSS. Where it is low, consideration may be given to strategies that boost self-efficacy, such as intensive wellness education ( $\mathrm{Ng}$ et al 2013). This is supported by previous suggestions that measuring and promoting self-efficacy may help increase exercise participation for individuals with MS (Smith 2012).

Theme two centred on goals and the purpose they give to Norman's PA engagement. For Norman, the goal of regaining his ability to walk gave him a strong purpose to pursue regular PA. While the role of ambitious goals in neurological rehabilitation is controversial, some clinicians believe that goals do not have to be achievable to be motivating (Playford et al. 2009). Indeed Norman's case provides evidence that, at least for some individuals, seemingly unrealistic goals can sustain PA motivation. The value of goals in Norman's case supports arguments made by Kosma et al (2012) that goal setting should form an important part of PA interventions. It is also consistent with Morrison and Stuifnbergen (2014), who found social and physical outcome expectations partly explained PA participation levels in individuals with long-standing MS (>15 years). Norman had experienced the symptoms of MS for 8 years when interviewed. Building on previous research, therefore, the present case suggests that goals or outcome expectations may also encourage PA participation in individuals with MS who do not meet long-standing criteria. In combination, these findings suggest it may be valuable for people with MS to establish positive goals or outcome expectations with their physiotherapists. In particular, our findings support the idea that programmes like Blue Prescription (Hale et al 2013) that are guided more by the goals of people with MS could provide a greater sense of purpose that motivates PA participation and adherence.

Norman saw PA as a necessity for achieving his "ultimate" goal of walking again (subtheme 2.1), giving him a strong motivation to engage in PA on a regular basis. Therefore, appraising PA as a necessity may be a deliberate cognitive strategy useful for enhancing PA participation in other individuals with MS. In particular, physiotherapists could consider encouraging people with MS to see PA as a necessary component of their well-being. However, such a strategy would likely require interdisciplinary commitment, given the different exercise advice individuals with MS may receive from different healthcare professionals (Smith et al 2013). Norman's goal of walking also kept him determined to engage in PA despite setbacks (subtheme 2.2). Thus physiotherapists may consider setting an overarching, purposeful goal with patients, such as working towards regaining a former capability or completing an appropriate physical event (e.g. a $5 \mathrm{~km}$ fun run). In turn, this may assist individuals with MS in garnering the motivation necessary to overcome PA barriers when setbacks are experienced.
The present study was limited by methodological constraints of carrying out a mixed methods case-study and the use of only one think-aloud interview with Norman. The think-aloud process is an established method of collecting qualitative data (Al-Janabi et al 2013, Anderson-Lister and Treharne 2014, Lundgrén-Laine and Salanterä 2010, Koro-Ljungberg et al 2013, McGavock and Treharne 2011) with some strengths and limitations. Think-aloud interviews are less like a natural conversation than semi-structured interviews and require considerable attention in answering the questionnaires as well as verbal questions, which can be fatiguing (although this was not the case for Norman). Think-aloud interviews are not necessarily any more leading than semi-structured interviews where interviewers often dominate the flow of the interaction with very detailed lists of questions (Willig, 2008). The benefit of think-aloud interviews is that they provide qualitative data directly relating to the questionnaire items and thus inform knowledge about key concepts covered by the questionnaires. The method also allows for probing verbal questions to be asked as in a semi-structured fashion but with less focus on pseudo-naturalistic conversational talk because the participant and researcher are oriented to talking about experiences prompted by the questionnaire items. Our use of both qualitative data and quantitative data (from the questionnaires) allowed for triangulation to create a more holistic understanding; however, mixed methods research and triangulation can result in the loss of some context-specific instances of the phenomena under investigation (Willig 2008). While every attempt was made to capture the nuances of Norman's experience, some of these may have been lost in making sense of his story as a whole. Furthermore, case studies are characterised by an idiographic or individual nature (Willig 2008). Thus Norman's experience may only transfer to some other individuals with MS. However, use of a single case approach has allowed us to present an in-depth account of Norman's thoughts related to PA motivation in the hope that thought processes useful in improving PA intervention strategies for the MS community might be revealed.

Future research could address whether the processes identified for Norman's case, such as using positivity to stay in control, using PA to generate positive self-talk and setting purposeful goals, would likely be considered helpful by other members of the MS community. In addition, future research could address whether goal setting underlies positivity and PA motivation. Studies would ideally include individuals of other genders as well as a range of ages and ethnicities. In addition, research into the efficacy of the aforementioned processes would help assess whether they are valuable elements to incorporate into physiotherapy interventions. Ultimately, such studies would further our understanding of whether thought processes and, in particular, positive thinking could enhance PA participation in the MS community.

In summary, there are several ways Norman's case may apply to physiotherapy interventions for people with MS. Physiotherapists may consider using thought strategies such as positive self-talk to attempt to foster positivity among people with MS. They may consider assessing self-efficacy and implementing strategies to boost self-efficacy where appropriate. People with MS may 
benefit from setting overarching, purposeful goals with the input of their physiotherapists to motivate PA engagement in the face of setbacks. Finally, physiotherapists may encourage patients to think about PA as a necessary element of their wellbeing. These considerations must be recognised as important aspects from one individual case of MS. As such, they may be useful for some individuals with MS and not for others. Nonetheless, the elements identified may be of benefit for improving future physiotherapy interventions for individuals with MS, particularly once their effectiveness for the wider MS community has been investigated.

\section{CONCLUSION}

In the present study we have presented a case of the thought processes and PA motivation of one individual, who is both particularly positive and motivated to engage in PA. For Norman, being positive is a choice which he argues helps him to cope with his MS and remain in control of his life. PA helps him to maintain this positive outlook. Goals give him a purpose for doing PA and provide determination to engage in PA on a regular basis. While it is too early to conclude these thought processes would enhance motivation in the MS community, Norman's case certainly raises this possibility. Furthermore, it justifies the need for further investigation into whether these thought processes might benefit physiotherapy interventions for individuals with MS.

\section{KEY POINTS}

1. Positive thinking and purposeful goals were central to high PA motivation in our case study of one individual with MS.

2. Physiotherapists may consider fostering positive thinking and purposeful goal setting to strengthen PA motivation in physiotherapy interventions for individuals with MS.

3. In particular, positive self-talk statements, self-efficacy assessment, setting overarching goals with MS patients and having them view PA as a necessary component of wellbeing could be useful as tools for physiotherapists.

4. Further research should test the relevance of positive thinking and purposeful goals to the wider MS community and their effectiveness in promoting PA participation.

\section{PERMISSIONS}

This study was approved by the University of Otago Human Ethics Committee (allocation number: DP29/11). The participant signed informed consent before taking part.

\section{DISCLOSURES}

This study was funded by the Department of Psychology at the University of Otago. The authors declare no conflict of interest.

\section{ADDRESS FOR CORRESPONDENCE}

Gareth J. Treharne, Department of Psychology, University of Otago, PO Box 56, Dunedin 9054, New Zealand. Telephone: 03 479 7630. Email: gareth.treharne@otago.ac.nz

\section{REFERENCES}

Al-Janabi H, Keeley T, Mitchell P, Coast J (2013) Can capabilities be selfreported? A think aloud study. Social Science and Medicine 87: 116-122.

Anderson-Lister G, Treharne GJ (2014) 'Healthy individuals' perceptions of type 1 and type 2 diabetes cause and management: A 'think-aloud,' mixed-methods study using video-based vignettes. Journal of Health Psychology 19(11): 1371-1381. doi: 10.1177/1359105313490315.

Bekhet AK, Zauszniewski JA (2013) Measuring use of positive thinking skills psychometric testing of a new scale. Western Journal of Nursing Research. 35(8): 1074-1093. doi: 10.1177/0193945913482191.

Braun V, Clarke V (2006) Using thematic analysis in psychology. Qualitative Research in Psychology 3(2): 77-101. doi:10.1191/1478088706qp063oa.

Braun V, Clarke V (2013) Successful qualitative research: A practical guide for beginners. London: Sage.

Chiu CY, Fitzgerald SD, Strand DM, Muller V, Brooks J, Chan F (2012) Motivational and volitional variables associated with stages of change for exercise in multiple sclerosis: a multiple discriminant analysis. Rehabilitation Counselling Bulletin 56(1): 23-33. doi: 10.1177/0034355212439898.

Dlugonski D, Joyce RJ, Motl RW (2012) Meanings, motivations and strategies for engaging in physical activity among women with multiple sclerosis. Disability and Rehabilitation 34(25): 2148-2157. doi: 10.3109/09638288.2012.677935.

French DP, Cooke R, Mclean N, Williams M, Sutton S (2007) What do people think about when they answer theory of planned behaviour questionnaires? A think aloud study. Journal of Health Psychology 12(4): 672-687. doi: 10.1177/1359105307078174.

Fonteyn ME, Kuipers B, Grobe SJ (1993) A description of think aloud method and protocol analysis. Qualitative Health Research 3(4): 430-441. doi: $10.1177 / 104973239300300403$.

Hale LA, Mulligan HF, Treharne GJ, Smith CM (2013) The feasibility and shortterm benefits of Blue Prescription: a novel intervention to enable physical activity for people with multiple sclerosis. Disability and Rehabilitation 35(14): 1213-1220. doi: 10.3109/09638288.2012.723787.

Hale LA, Smith C, Mulligan H, Treharne GJ (2012) "Tell me what you want, what you really really want...": Asking people with multiple sclerosis about enhancing their participation in physical activity. Disability and Rehabilitation 34(22): 1887-1893. doi: 10.3109/09638288.2012.670037.

Hall-McMaster SM, Treharne GJ, Smith CM (2015) 'The positive feel': Unpacking the role of positive thinking in people with multiple sclerosis's thinking aloud about staying physically active. Journal of Health Psychology, Published online before print July 13, 2015, doi: 10.1177/1359105315592047.

Kasser S (2009) Exercising with multiple sclerosis: Insights into meaning and motivation. Adapted Physical Activity Quarterly 26(3): 274-289.

Koro-Ljungberg M, Douglas EP, McNeill N, Therriault D (2013) Reconceptualizing and de-centering think-aloud methodology in qualitative research. Qualitative Research 13(6): 735-753. doi: 10.1177/1468794112455040.

Kosma M, Ellis R, Bauer JJ (2012) Longitudinal changes in psychosocial constructs and physical activity among adults with physical disabilities. Disability and Health Journal 5(1): 1-8

Learmonth YC, Marshall-McKenna R, Paul L, Mattison P, Miller L (2013) A qualitative exploration of the impact of a 12-week group exercise class for those moderately affected with multiple sclerosis. Disability and Rehabilitation 35(1): 81-88. doi: 10.3109/09638288.2012.688922.

Lundgrén-Laine H,Salanterä S (2010) Think-aloud technique and protocol analysis in clinical decision-making research. Qualitative Health Research 20(4): 565-575. doi: 10.1177/1049732309354278.

McGavock ZC, Treharne GJ (2011) Young adults' beliefs about people living with HIVIAIDS and rheumatoid arthritis: thematic analysis of a think-aloud questionnaire investigation. New Zealand Journal of Psychology 40(3): 71-78. 
Morrison, JD, Stuifbergen AK (2014) Outcome expectations and physical activity in persons with longstanding multiple sclerosis. Journal of Neuroscience Nursing 46(3): 171-179. doi: 10.1097/ JNN.0000000000000050.

Motl RW, McAuley E, Snook EM (2005) Physical activity and multiple sclerosis: a meta-analysis. Multiple Sclerosis 11(14): 459-463. doi: 10.1191/1352458505ms1188oa.

Ng A, Kennedy P, Hutchinson B, Ingram A, Vondrell S, Goodman T, Miller D (2013) Self-efficacy and health status improve after a wellness program in persons with multiple sclerosis. Disability and rehabilitation 35(11), 10391044. doi: 10.3109/09638288.2012.717586

Playford ED, Siegert R, Levack W, Freeman J (2009) Areas of consensus and controversy about goal setting in rehabilitation: a conference report Clinical Rehabilitation 23(4): 334-344. doi: 10.1177/0269215509103506.

Plotnikoff RC, Lippke S, Reinbold-Matthews M, Courneya KS, Karunamuni N, Sigal RJ, Birkett N (2007) Assessing the validity of a stage measure on physical activity in a population-based sample of individuals with Type 1 or Type 2 diabetes. Measurement in Physical Education and Exercise Science 11(2): 73-91. doi: 10.1080/10913670701294062.

Radley A, Chamberlain K (2001) Health psychology and the study of the case: from method to analytic concern. Social Science \& Medicine 53(3): 321332.

Rigby SA, Domenech C, Thornton EW, Tedman S, Young CA (2003) Development and validation of a self-efficacy measure for people with multiple sclerosis: The Multiple Sclerosis Self-efficacy Scale. Multiple Sclerosis 9(1): 73-81. doi: 10.1191/1352458503ms870oa.

Roger K, Wetzel M, Hutchinson S, Packer T, Versnel, J (2014) "How can I still be me?": Strategies to maintain a sense of self in the context of a neurological condition. International Journal of Qualitative Studies on Health and Well-Being 9. doi: 10.3402/qhw.v9.23534.

Smith C (2012) Exercise: 'Friend or foe' for people with multiple sclerosis who experience fatigue? New Zealand Journal of Physiotherapy 40(1): 29-32.
Smith CM, Hale LA, Olson K, Baxter GD, Schneiders AG (2013) Healthcare provider beliefs about exercise and fatigue in people with multiple sclerosis. Journal of Rehabilitation Research and Development 50(5): 733744. doi: 10.1682/JRRD.2012.01.0012.

Smith CM, Fitzgerald HJM, Whitehead L (2014) How fatigue influences exercise participation in men with multiple sclerosis. Qualitative Health Research. 25(2):179-188. doi: 10.1177/1049732314551989.

Smith C, Olson K, Hale LA, Baxter D, Schneiders AG (2011) How does fatigue influence community-based exercise participation in people with multiple sclerosis? Disability \& Rehabilitation 33(22-23): 2362-2371. doi: 10.3109/09638288.2011.573054.

Stroud NM, Minahan CL (2009) The impact of regular physical activity on fatigue, depression and quality of life in persons with multiple sclerosis. Health and Quality Life Outcomes 7(1): 68-69. doi: 10.1186/1477-75257-68.

Thomas G (2010) Doing case study: abduction not induction, phronesis not theory. Qualitative Inquiry 16(7): 575-582. doi: 10.1177/1077800410372601.

Treharne GJ, Riggs DW (2014) Ensuring quality in qualitative research. In P. Rohleder \& A. C. Lyons (Eds.), Qualitative Research in Clinical and Health Psychology (pp. 57-73). Basingstoke: Palgrave MacMillan.

Van Oort LC, Schröder C, French DP (2011) What do people think about when they answer the Brief Illness Perception Questionnaire? A 'thinkaloud' study. British Journal of Health Psychology 16(2): 231-245. doi: 10.1348/135910710X500819.

Wicks P, Vaughan TE, Massagli MP (2012) The multiple sclerosis rating scale, revised (MSRS-R): Development, refinement, and psychometric validation using an online community. Health and Quality of Life Outcomes 10(70). doi: 10.1186/1477-7525-10-70.

Willig C (2013) Introducing qualitative research in psychology ( $3^{\text {rd }}$ edn). Berkshire: Open University Press. 